\title{
An Artificial Neural Network-Based Method for Railway Logistics Network Design
}

\author{
Wang Danzhu, ${ }^{1, a}$, Lang Maoxiang ${ }^{1, b}$ \\ ${ }^{1}$ Beijing Jiaotong University, Beijing, 100044, China \\ âwangdanzhu1985@sohu.com, ${ }^{b}$ mxlang@bjtu.edu.cn
}

\begin{abstract}
Keywords: railway, logistics network design, Self-Organizing Map neural network, BP neural network.
\end{abstract}

\begin{abstract}
Logistics network design problem has an important position in the railway logistics development, it has aroused great concern both in railway transportation and logistics research fields. This paper proposes a method for railway logistics network design problem based on artificial neural network model. In the logistics network design method, various influencing factors of railway logistics network have been considered. An evaluation index system of a railway transportation enterprise is set up. Self-Organizing Map neural network algorithm has been used for the creation of logistics network nodes initial set. And the layers division of the logistics network has been determined with the help of BP neural network model. Subsequently an empirical study of a railway logistics enterprise is given to certificate the feasibility and accuracy of this railway logistics network design method.
\end{abstract}

\section{Introduction}

Logistics network design problem covers a wide range of subjects, such as economics, politics, mathematics, ecology and so on. Therefore, many influencing factors should be considered during the process of logistics network planning, and there are a variety of methods to establish a logistics network as well. The related work about logistics network design methods of manufacture enterprises, commercial enterprises and third-party logistics enterprises has been achieved fruitful results, but the research on railway logistics network design methods still has a long way to go. Especially, with the rapid development of Chinese economy, railway transportation market demand has been turned into all-around logistics service demand. It is high time that railway enterprises should establish their own logistics network, or they will be at a disadvantage in the fierce competition of the logistics market.

There are a certain number of papers about logistics network design methods. Some examples can be given as follows: Jean-Francois Cordeau et al. [1] focused on an iterated local search heuristic for the logistics network design problem with single assignment, evaluated the performance of the algorithm on randomly generated instances. Suh-Wen Chiou et al. [2] proposed a bi-level programming for logistics network design with system-optimized flows, presented a novel solution algorithm to efficiently solve the logistics network design problem. Phuong Nga Thanh et al. [3] investigated a linear relaxation-based heuristic approach for logistics network design, and planning a multi-period, multi-echelon, multi-commodity logistics network with deterministic demands. Hüseyin Basligil, Selin Soner Kara et al. [4] discussed the distribution network optimization problem for third party logistics service providers, and developed a new methodology to determine optimal routes for vehicles with different capacities and different costs for logistics companies. Mir Saman Pishvaee et al. [5] presented a memetic algorithm for bi-objective integrated forward/ reverse logistics network design, proposed a model for integrated logistics network design to avoid the sub-optimality caused by a separate, sequential design of forward and reverse logistics networks.

Overall, substantial achievements have been gained in this research fields, and the theory and application of logistics network design methods has been tend to mature [6]. Thence, all of these works laid a solid foundation for future research in this paper. 


\section{Model Construction}

Characteristics of Railway Logistics Network. Railway logistics network is different from other logistics networks, and it has its own characteristics. One of the most distinctive characteristic of railway logistics network is that railway logistics network is based on railway transportation. Second, railway logistics network design is always implemented by governments or large railway transportation enterprises whose business scope spreads the whole country. Hence, railway logistics network design usually is a macro planning problem. At last, in railway logistics network design problem, sometimes, the logistics market demand is unspecific [7]. In other words, railway logistics network design must be carried out under fuzzy demand environment [8]. In this sense, traditional logistics network design methods based on logistics nodes location models are not applicable.

To tackle this problem, the paper proposes a fuzzy demand-oriented logistics network design method based on Artificial Neural Network, and realizes the method by two models: the logistics nodes initial set determination model and the logistics network level division model.

The Process of Railway Logistics Network Design. In the railway logistics network design model, a three-layer network will be established by the following steps:

Step 1. Establishing an evaluation index system of railway logistics nodes characteristics, which is a basis of the Self-Organizing Map neural network model and BP neural network model.

Step 2. Finding out all the optional railway logistics nodes in the service range of the railway logistic network. Denoted by a set of $\varphi$.

Step 3. Determining the initial set of the railway logistics nodes included in the logistics network, using self-organizing map neural network model. Denoted by a set of $\varphi^{*}$, where $\varphi^{*} \subseteq \varphi$.

Step 4. Dividing the initial set of railway logistics nodes into three classifications, and each classification represents a layer of the logistics network. Logistics nodes which belong to layer I, means they are in the highest level of the logistics network and play the most important role of the network operation. In this process, we use a BP neural network model.

Step 5. If necessary, make an adjustment and optimization of the railway logistics network, according to the experience of logistics network designers and managers.

We consider the railway logistics network composed of three layers, like the one depicted in Fig. 1.

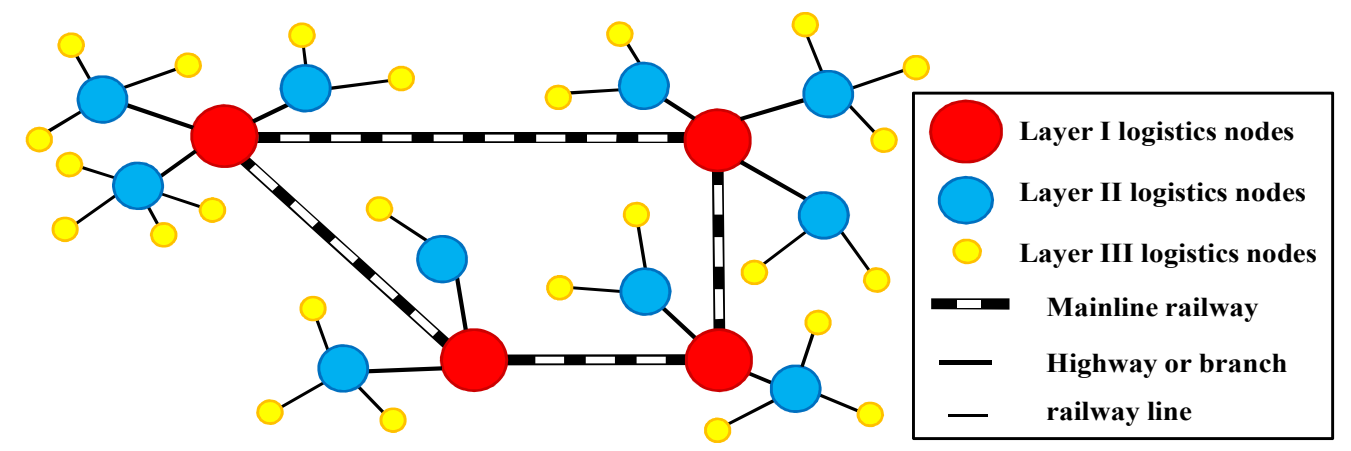

Figure 1. A three-layer railway logistics network

\section{An Initial Set Determined Model}

Railway Logistics Nodes Evaluation Index System. There are various kinds of influencing factors of logistics nodes should be considered in the railway logistics network design, so specific evaluation index system should be established in different logistics network design projects under current situation. In this paper, an evaluation index system of a nationwide railway material circulation enterprise is taken as an example. The main business of this enterprise is transportation and logistics services of railway materials and steel. So, the 
influence of gross industrial production and steel consumption must be considered in the evaluation index system. A three-layer evaluation index system is established to represent the influencing factors of the logistics nodes. Table 1 gives the results of the evaluation index system. In the following research of this paper, the factors of layer 3 will be used in the models and denoted by $x_{1}, x_{2} \ldots x_{12}$.

Table 1. Evaluation index system

\begin{tabular}{l|c|c}
\hline Layer 1 factors & Layer 2 factors & Layer 3 factors \\
\hline \multirow{4}{*}{$\begin{array}{l}\text { Enterprise internal } \\
\text { influencing factors }\end{array}$} & Existing logistics demand & Storage resources $\left(x_{1}\right)$ \\
\cline { 2 - 3 } & & Railway transport logistics scale $\left(x_{2}\right)$ \\
\cline { 2 - 3 } & Potential logistics demand & $\begin{array}{r}\text { Railway transportation service } \\
\text { categories }\left(x_{3}\right)\end{array}$ \\
\cline { 2 - 3 } & Enterprise internal policies & Potential logistics demand scale $\left(x_{4}\right)$ \\
\cline { 2 - 3 } & Economic factors & Strategic partners $\left(x_{6}\right)$ \\
\cline { 2 - 3 } Enterprise external & Gross domestic product $\left(x_{7}\right)$ \\
\cline { 2 - 3 } influencing factors & Main business related factors & Gross industrial production $\left(x_{8}\right)$ \\
\cline { 2 - 3 } & Traffic location & Steel consumption $\left(x_{9}\right)$ \\
\cline { 2 - 3 } & National policies & Port throughput $\left(x_{10}\right)$ \\
\cline { 2 - 3 } & & Traffic convenience $\left(x_{11}\right)$ \\
\hline
\end{tabular}

The units of the factors from $x_{1}$ to $x_{12}$ are: ten thousand square meters, ten thousand tons, none, ten thousand tons, none, none, a hundred million yuan, a hundred million yuan, ton, none and none.

Self-Organizing Map Neural Network. Self-Organizing Map (SOM) is a type of artificial neural network which is trained by an unsupervised learning method. It produces a lowdimensional (typically two-dimensional) map to represent the discretized representation of the input space of the training samples. The model established in this section is shown in Fig. 2.

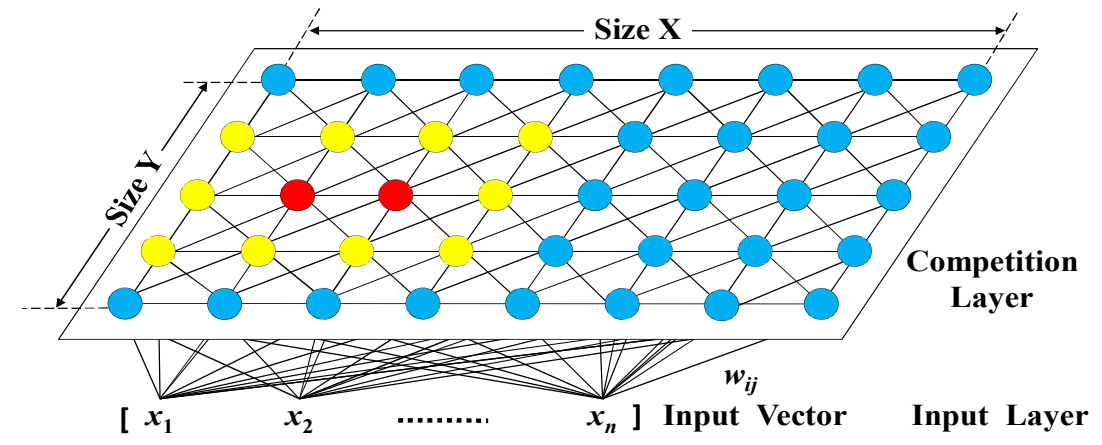

Figure 2. Topological structure of the SOM neural network model

SOM neural network learning algorithm includes three processes: competitive process, cooperative process and renewal process.

\section{(1) Competitive process}

In competitive process, neurons that have the maximum output are defined as the winning neurons. As a result of the linear activation function of neurons, the maximum output depends on the input of a neuron $u^{i}=\sum_{j=1}^{N} w_{i j} x_{j}$, that is, the inner product of input vector $\boldsymbol{X}=\left(x_{1}, x_{2}, \cdots, x_{n}\right)^{\mathrm{T}}$ and weight vector $\boldsymbol{W}=\left(w_{i 1}, w_{i 2}, \cdots, w_{i N}\right)^{\mathrm{T}}, i=1,2, \cdots, M$. When the input vector and the weight vector are normalized vectors, this inner product is equivalent to the minimum Euclidean distance between the input vector and the weight vector. In mathematics, the Euclidean distance is the "ordinary" distance between $\boldsymbol{Y}=\left(y_{1}, y_{2}, \cdots, y_{N}\right)^{\mathrm{T}}$ and $\boldsymbol{Z}=\left(z_{1}, z_{2}, \cdots, z_{N}\right)^{\mathrm{T}}$ in Euclidean space. 
The value of the Euclidean distance is $\sum_{i=1}^{N}\left(y_{i}-z_{i}\right)^{2}$. Therefore, when the input vector is $X$ and the number $c$ neuron is the winning neuron, which satisfies condition [9]:

$$
\left\|\boldsymbol{X}-\boldsymbol{W}_{c}\right\|=\min \left\|X-W_{i}\right\|, \quad i=1,2, \cdots M
$$

where $\|\bullet\|$ denotes the Euclidean distance between the input vector $\boldsymbol{X}$ and the weight vector $\boldsymbol{W}_{\boldsymbol{i}}$.

(2) Cooperative process

In the cooperative process, the strength center of winning neurons will be found out. The center of topology neighborhood is the winner of the competitive neurons. The neuron within the neighborhood will be called as excitatory neuron, which is strength center.

(3)Renewal process

During the renewal process, improved Hebbian learning rule will be used to update the weight vector between neurons which is within the winning neuron topology neighborhood of the neural network.

Self-Organizing Map Neural Network Modeling. The values of influencing factors in the evaluation index system are used as input vectors of the SOM neural network model, denoted by $\boldsymbol{X}=\left(x_{1}, x_{2}, \cdots, x_{12}\right)^{\mathrm{T}}$. And the steps of SOM learning algorithm of this model are as follows:

Step 1. Initialization. Randomize the weight vectors between input neurons and output neurons.

Step 2. Grab an input vector $X$.

Step 3. Calculate the Euclidean distance $d_{j}$ between each input samples and output neuron $j$ :

$$
d_{j}=\left\|X-W_{j}\right\|=\sqrt{\sum_{i=1}^{N}\left[x_{i}(t)-w_{i j}(t)\right]^{2}}
$$

Find out a neuron $j^{*}$ which has the minimum distance, and determine a unit $k$ :

$$
d_{k}=\min _{j}\left(d_{j}\right), \quad \forall j
$$

Step 4. Give a neigtborhood: $S k(t)$.

Adjust the weight of output neuron $j^{*}$ and its "adjacency neurons":

$$
w_{i j}(t+1)=w_{i j}(t)+\eta(t)\left[x_{i}(t)-w_{i j}(t)\right]
$$

where $\eta$ is a gain item, and decreases with time until it eventually reaches zero. Usually, $\eta(t)=1 / t$.

Step 5. Caculate the output $o_{k}$ :

$$
o_{k}=f\left(\min _{j}\left\|X-W_{j}\right\|\right)
$$

where $f=\left\{\begin{array}{l}1 \\ 0\end{array}\right.$, or other nonlinear functions.

Step 6. Select new learning samples, repeat from step2 until the map has converged or predefined no. of training cycles have passed [10].

The initial set of the railway logistics nodes included in the logistics network $\varphi^{*}$ can be found out by this model, and the initial set $\varphi^{*}$ will be a basis of the railway logistics network layers classification model based on BP neural network algorithm.

\section{A Railway Logistics Network Layers Classification Model}

After determining the initial set of the logistics nodes, three layers must be classified in the railway logistics network. A supervised BP neural network algorithm is used in this part.

BP Neural Network. BP neural network is another type of artificial neural network that is trained by a supervised learning method, called back-propagation algorithm. It has a layered structure, and each layer consists of units which receive their inputs from a layer directly above 
and send their outputs to units in a layer directly below the units[11]. There are no connections within a layer. The topological structure of the BP neural network model used in this paper is like the one depicted in Fig. 3.

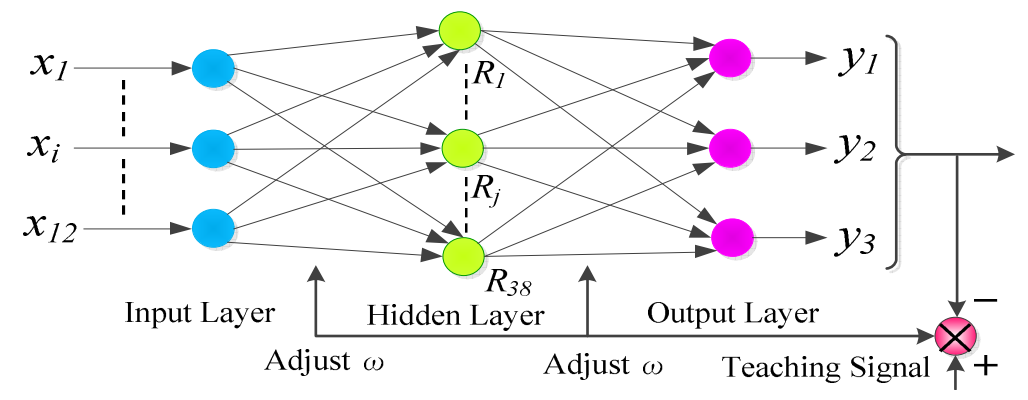

Figure 3. Topological structure of the BP neural network model

BP Neural Network Modeling. The BP neural network modeling here is a multi-layer feedforward network, and it will be trained by the back-propagation learning method. Define the error function of this network as:

$$
E=\frac{1}{2} \sum_{i}\left(O_{i}^{m}-Y_{i}^{m}\right)^{2}
$$

where $E$ denotes the system error of the network; $Y_{i}^{m}$ denotes the expected output of the output unit (or neuron) $i$ of the layer $m$, also as a teaching signal; $O_{i}{ }^{m}$ denotes the actual output of the output unit (or neuron) $i$ of the layer $m$.

Considering that there are $n$ neurons in each layer, that is $i=1,2, \cdots, n ; j=1,2, \cdots, n$. To the neuron $i$ of the layer $k$, there are $n$ weights $\omega_{i 1}, \omega_{i 2}, \cdots, \omega_{i n}$. And using $\omega_{i n+1}$ as threshold, denoted by $\theta_{i}$. The input vector is $X=\left(x_{1}, x_{2}, \cdots, x_{12}\right)^{\mathrm{T}}$. When the activation function of the network chooses sigmoid function, the recursive solving method of the weights $\omega_{i j}$ involves the following steps:

Step 1. Set initial values of the weights $\omega_{i j}$.

Set a small nonzero random number (usually between 0 and 1 ) to every weight $\omega_{i j}$ as its initial value. Where $\omega_{i j}$ denotes the weight between neuron $i$ and neuron $j ; \omega_{i n+1}=-\theta ; \theta$ denotes the threshold of a neuron which belongs to the hidden layer or the output layer.

Step 2. Select a set of standardization training samples $X=\left(x_{1}, x_{2}, \cdots, x_{n}\right)^{\mathrm{T}}$, and expected output $Y=\left(y_{1}, y_{2}, \cdots, y_{n}\right)^{\mathrm{T}}$.

Step 3. Caculate the output $O$ of each layer.

The output vector $O_{i}^{k}$ of the neuron $i$ in layer $k$, subjected to:

$$
\begin{gathered}
n e t_{i}^{k}=\sum_{j=1}^{n+1} \omega_{i j} O_{j}^{k-1} \\
O_{i}^{k}=f\left(n e t_{i}^{k}\right)
\end{gathered}
$$

where $n e t_{i}^{k}$ denotes the output of neuron $i$ in layer $k ; f(x)$ is a nonlinear differentiable nondecreasing function. In this model, select sigmoid function as $f(x)$, thus $f(x)=1 / 1+e^{-x}$; $O_{n+1}^{k-1}=1 ; \omega_{i n+1}=-\theta$.

Step 4. Caculate the learning error of each layer.

To the output layer, $k=m$, that is: $d_{i}^{k}=O_{i}^{k}\left(1-O_{i}^{k}\right)\left(O_{i}^{k}-Y_{i}\right)$.

To other layers, that is: $d_{i}^{k}=O_{i}^{k}\left(1-O_{i}^{k}\right) \sum_{l} \omega_{l i} d_{i}^{k+1}$, where $l=1,2, \cdots, i$.

Step 5. Adjust the weights $\omega_{i j}$.

Considering the traditional back-propagation learning method only adjusts the weights along the gradient direction, and has slow convergence speed. So a momentum back-propagation algorithm is used in this study. The weights adjustment rules of this method are: 


$$
\omega(t+1)=\omega(t)+\lambda[(1-\sigma) D(t)+\sigma D(t-1)]
$$

where $D(t)=-\frac{\partial E}{\partial \omega(t)}$ denotes the negative gradient at time $t ; D(t-1)$ denotes the negative gradient at time $t-1 ; \lambda$ denotes learning rate, $0<\lambda<1 ; \sigma$ denotes momentum factor, always between $0.1 \sim 0.8$.

The self-adaptive adjustment method of learning rate is: $\alpha(\lambda)=2^{\lambda} /(t-1)$, where $\lambda=\operatorname{sign}[D(t) D(t-1)]$.

If after two successive iterations, the gradient has the same direction, means the convergence speed is slow. Therefore, the learning rate should be doubled. Otherwise, if the convergence speed is excessive, the learning rate should be halved.

Step 6. After caculating all the weights of each layer, the system error should be checked. If the system error Eq. 6 meets the qualification, the learning method can stop. Otherwise, repeat from step 3 until the system error meets the qualificaton.

A three-layer railway logistics network will be established by this BP neural network model, if necessary, the optimization and adjustment of the logistics network can be given by the experts and managers, according to their research experience and management experience.

\section{Empirical Study}

A numerical example is solved to represent the efficiency of our methodology. The railway logistics network design model is used for designing a logistics network of a railway transportation enterprise.

Through investigating, the basic data about logistics operation of the enterprise was collected. The firsthand statistics data was gathered up from various sources, including 163 cities information in China. Each of them is considered as a reserve logistics node, and all of them compose the initial set of logistics nodes $\varphi$, which we referred in section II. Every logistics node has 12 factors (see Table 1). However, in the view of the fact that page layout is limited, the original data can't be shown here.

Data Preprocessing. The values of influencing factors have different units, and the value size between the maximum value and the minimum value has an obvious difference. So, the normalization processing should be taken to the initial data. In order to improve the operation precision of the SOM neural network model and the BP neural network model, the initial data is normalized into [0.05, 0.95].

Input and Output Design. After the normalization processing, the input and output vectors will be designed in this section. The 12 influencing factors $x_{1}$ to $x_{12}$ are designed as the input vectors, denoted by $X^{\prime}=\left(x_{1}, x_{2}, \cdots, x_{12}\right)^{\mathrm{T}}$ (after normalization).

The SOM neural network model uses the unsupervised learning method, so the expected output vectors are unnecessary. The outputs of SOM model are different clusters of the logistics node.

The BP neural network model is trained by a supervised learning method, so we must design the expected output vectors as teaching signals. The railway logistics network of the enterprise is divided into three layers: layer I, layer II and layer III. Therefore, the output of this BP model should be designed as $Y_{1}, Y_{2}$ and $Y_{3}$. $Y_{1}$ represents the logistics node belongs to layer I; $Y_{2}$ indicates the logistics node belongs to layer II; $Y_{3}$ means the logistics node belongs to layer III.

In the output vector $Y=\left(y_{1}, y_{2}, y_{3}\right)^{\mathrm{T}}, y_{i}(i=1,2,3)$ is the membership degree of this sample belongs to the layer. After calculating the vector $Y$, we will decide the logistics node belongs to which layer by the maximum membership degree law. For example, the output of the model is $(0.0011,0.0036,0.9734)$, means this logistics node belongs to the layer III, because the last component of the output vector is the maximum. Then, we record this output vector as $(0,0,1)$. Numerical Calculation and Model Simulation. As the design of this model is completed, a simulation will be implemented based on Matlab 7.0 software platform. 


\section{(1) Simulation of the SOM model}

The initial data set $\varphi$ includes 163 records (after normalization), taking all these records as training samples, and the train epochs is 200 . The cluster result is depicted in Table 2.

Table 2. The cluster result of SOM model simulation

\begin{tabular}{c|c|c|c|c|c|c|c|c|c|c}
\hline No. & $\mathbf{1}$ & $\mathbf{2}$ & $\mathbf{3}$ & $\mathbf{4}$ & $\mathbf{5}$ & $\mathbf{6}$ & $\mathbf{7}$ & $\mathbf{8}$ & $\mathbf{9}$ & $\mathbf{1 0}$ \\
\hline Total number of nodes & 14 & 5 & 11 & 15 & 19 & 20 & 7 & 10 & 29 & 33 \\
\hline
\end{tabular}

The SOM model divides all the reserve logistics nodes into 10 clusters. Comprehensive consideration based on the actual situation of the enterprise and the railway logistics network scale, the logistics nodes belong to clusters 2, 3, 5, 6, 7, 9 and 10 will be included in the final logistics network [12], and the nodes belong to clusters 1, 4 and 8 will not be included (39 nodes). Consequently, the final set of logistics nodes $\varphi^{*}$ includes 124 elements, you can find it in Table 3 (including all the cities in Table 3 ).

\section{(2) Simulation of the BP neural network model}

BP neural network model is used to divide the layers of the logistics network. We choose 30 records as training samples: 11 of them belongs to layer I; 14 of them belongs to layer II; 5 of them belongs to layer III. Based on experience and test, when there are 38 neurons in the hidden layer, the BP neural network model can get the most satisfactory result. The maximum training times is 10000 ; the minimum mean square error is $1 * \mathrm{e}^{-5}$.

The simulation result of this model is: there are 18 logistics nodes in the layer I; 41 logistics nodes in layer II; 65 logistics nodes in layer III (see Table 3).

Table 3. Simulation result of BP Neural Network model

\begin{tabular}{|c|c|}
\hline Layer & Logistics Node Cities Name \\
\hline $\begin{array}{c}\text { Layer I } \\
(18 \text { cities })\end{array}$ & $\begin{array}{c}\text { Beijing, Tianjin, Tangshan, Taiyuan, Baotou, Shenyang, Harbin, Shanghai, Wuxi, Qingdao, Rizhao, } \\
\text { Zhengzhou, Wuhan, Guangzhou, Nanning, Xian, Chengdu and Kunming }\end{array}$ \\
\hline $\begin{array}{l}\text { Layer II } \\
\text { (41 cities) }\end{array}$ & $\begin{array}{c}\text { Shijiazhuang, Qinhuangdao, Handan, Cangzhou, Datong, Yuncheng, Hohhot, Dalian, Dandong, } \\
\text { Jinzhou, Yingkou, Changchun, Jilin, Mudanjiang, Nanjing, Lianyungang, Suzhou, Hangzhou, } \\
\text { Ningbo, Hefei, Ma On Shan, Chaohu, Fuzhou, Xiamen, Nanchang, Ganzhou, Jinan, Pingdingshan, } \\
\text { Changsha, Shenzhen, Zhanjiang, Foshan, Fangchenggang, Liuzhou, Haikou, Lanzhou, Xining, } \\
\text { Yinchuan, Urumqi, Chongqing and Guiyang }\end{array}$ \\
\hline $\begin{array}{l}\text { Layer III } \\
(65 \text { cities })\end{array}$ & $\begin{array}{l}\text { Baoding, Langfang, Changzhi, Qinzhou, Shuozhou, Ordos, Tongliao, Anshan, Benxi, Xilin Gol } \\
\text { League, Alxa League, Fushun, Liaoyang, Daqing, Baicheng, Jixi, Xuzhou, Nantong, Changzhou, } \\
\text { Yancheng, Yangzhou, Zhenjiang, Wenzhou, Shaoxing, Taizhou, Yingtan, Jiaxing, Wuhu, Huzhou, } \\
\text { Quanzhou, Longyan, Weihai, Binzhou, Zibo, Weifang, Yantai, Taian, Dongying, Liaocheng, Linqin, } \\
\text { Laiwu, Dezhou, Jining, Qinzhou, Zaozhuang, Jiaozuo, Xuchang, Yichang, Huanggang, Zhuzhou, } \\
\text { Xiangtan, Dongguan, Maoming, Huizhou, Tianshui, Jiangmen Xieyang, Chongzuo, Baise, Weinan, } \\
\text { Baoji, Meishan, Yibin, Liupanshui and Qujing }\end{array}$ \\
\hline
\end{tabular}

\section{Results and Conclusion}

In this study, a new methodology of railway logistics network design is developed based on Artificial Neural Network. This method is different from traditional logistics node location model for it is oriented to the unspecific customer demand. Especially, it is suitable for macroplan of railway logistics network. A Self-Organizing Map neural network model and a BP neural network model are used for logistics network design, and a three-layer railway logistics network of the railway enterprise is established in the paper.

The most obvious advantage of integrated application of Self-Organizing Map neural network and BP neural network is: this method can combine the self-organizing characteristic base on the initial data' mathematical features of the SOM neural network with the goal-oriented supervised learning capacity using teaching signals of the BP neural network. It is seen that, this logistics network design method has a high economic and social benefit, and it will provide a reference for government decision-makers and enterprise managers. Although this method is deliberated, it maybe still has some disadvantages, further study is required. 


\section{References}

[1] Jean Francois Cordeau, Gilbert Laporte, Federico Pasin, An iterated local search heuristic for the logistics network design problem with single assignment, Int. J.Production Economics, 113 (2008)626-640.

[2] Suh-Wen Chiou, A bi-level programming for logistics network design with systemoptimized flows, Information Sciences, 179(2009)2434-2441.

[3] Phuong Nga Thanh, Olivier Peton, Nathalie Bostel, A linear relaxation-based heuristic approach for logistics network design, Computers \& Industrial Engineering, 59 (2010)964975.

[4] Huseyin Basligil,Selin Soner Kara,Pelin Alcan,Betul Ozkan,E.Gozde Caglar,A distribution network optimization problem for third party logistics service providers, Expert Systems with Applications, 38 (2011)12730-12738.

[5] Mir Saman Pishvaee, Reza Zanjirani Farahani, Wout Dullaert, A memetic algorithm for biobjective integrated forward/reverse logistics network design, Computers \& Operations Research, 37 (2010)1100-1112.

[6] Mir Saman Pishvaee, Fariborz Jolai,Jafar Razmi, A stochastic optimization model for integrated forward/reverse logistics network design, Journal of Manufacturing Systems, 28 (2009)107-114.

[7] Dameng Dai, Wu Chengwen, The study of individuation products customization systems based on Wiki, 2010 Third International Conference on Knowledge Discovery and Data Mining, (2010)560-563.

[8] Zhongfeng Qin, Xiaoyu Ji, Logistics network design for product recovery in fuzzy environment, European Journal of Operational Research, 202 (2010)479-490.

[9] Alex Seret, Thomas Verbraken, Sebastien Versailles, Bart Baesens, A new SOM-based method for profile generation: Theory and an application in direct marketing, European Journal of Operational Research, 220 (2012)199-209.

[10]Dr.Manu Pratap Singh, Dr.K.V.Arya, Ms.Kajal Sharma, Video Compression using Self Organizing Map and pattern storage using Hopfield Neural Network, Fourth International Conference on Industrial and Information Systems, 28-31 (2009)272-278.

[11]Fei Yin, Huajie Mao, Lin Hua, Zhiqiang Gu, Back propagation neural network based calculation model for predicting wear of fine-blanking die during its whole lifetime, Computational Materials Science, 59 (2012)140-151.

[12]Ajit K.Sahoo, Ming J.Zuo, M.K.Tiwari, A data clustering algorithm for stratified data partitioning in artificial neural network, Expert Systems with Applications, 39 (2012)70047014. 